\title{
Derivation of human embryonic stem cells at the Center of Regenerative Medicine in Barcelona
}

\author{
Begoña Aran • Ignasi Rodríguez-Pizà • Ángel Raya • \\ Antonella Consiglio • Yolanda Muñoz • Pere N. Barri • \\ Juan Carlos Izpisúa • Anna Veiga
}

Received: 6 April 2009 / Accepted: 14 January 2010 / Published online: 23 February 2010 / Editor: P. Andrews

(C) The Society for In Vitro Biology 2010

\begin{abstract}
We report here the legislative issues related to embryo research and human embryonic stem cell (hESC) research in Spain and the derivation of nine hESC lines at the Center of Regenerative Medicine in Barcelona. You can find the information for obtaining our lines for research purposes at blc@cmrb.eu.
\end{abstract}

B. Aran and I. Rodríguez-Pizà contributed equally to this work.

B. Aran · I. Rodríguez-Pizà · Á. Raya • A. Consiglio • Y. Muñoz • J. C. Izpisúa $\cdot A$. Veiga $(\bowtie)$

Center for Regenerative Medicine in Barcelona,

Barcelona, Spain

e-mail: aveiga@cmrb.eu

Á. Raya

Institució Catalana de Recerca i Estudis Avançats (ICREA),

Barcelona, Spain

\section{Á. Raya}

Networking Center of Biomedical Research in Bioengineering,

Biomaterials and Nanomedicine (CIBER-BBN),

Barcelona, Spain

\section{A. Consiglio}

Department of Biomedical Sciences and Biotechnology,

Unit of Biochemistry, University of Brescia,

25123 Brescia, Italy

P. N. Barri · A. Veiga

Department of Obstetrics, Gynecology and Reproduction,

Institut Universitary Dexeus,

Barcelona, Spain

J. C. Izpisúa

Gene Expression Laboratory, Salk Institute for Biological Studies,

La Jolla, CA 92037, USA
Keywords Embryo research legislation in Spain . $\mathrm{hESC}$ derivation

\section{Introduction}

Human embryo research legislation in Spain. In Spain, the possibility of conducting research with viable human embryos is a recent option; hence, there is limited experience compared with other countries. Such research became possible at the end of 2003 , when the implementation of Ley 45/2003 allowed the use of existing surplus frozen embryos for this purpose. Human embryos began to be frozen in Spain during the 1980s, and it is estimated that there are currently more than 50,000 such embryos in storage. With the recent passing of the new law on Assisted Reproduction Techniques (Ley 14/2006), any couple with frozen embryos, regardless of the time of cryopreservation, can decide on the future of their embryos from among four options: use by the couple for reproduction, donation to other couples, donation for research purposes, or disposal without further use. The new legislation allows couples to keep embryos frozen throughout the woman's reproductive life without establishing time limits. The Spanish Law on Biomedical Research from 2007 (Ley 14/2007, Ley de Investigación Biomédica) prohibits the creation of embryos for research purposes. However, it allows the production of human embryonic stem cell (hESC) for research or therapy using all the currently available technologies such as reprogramming of somatic cells through nuclear transfer and any other future technique (Article 33 of Ley de Investigación Biomedica). 
The legislation changes yielded to the creation of the National Stem Cell Bank (BNLC), with nodes in three separate locations (Barcelona, Valencia, and Granada), with the aim of guaranteeing availability and exchange of hESC lines (Orden SCO/393/2006). The BNLC promotes the quality and safety of the procedures and keeps personal and other ethically sensitive data confidential. Embryo donation cannot be reimbursed. Patient's informed consent is needed prior to the use of the embryos for derivation, and the name of the project must appear in the consent form. The approval of the corresponding Ethics Board is also essential, and the accrediting competent authority (Comisión de Seguimiento y Control de la Donación de Células y Tejidos Humanos del Instituto de Salud Carlos III) must approve the project.

We describe here the derivation methodology and the results obtained in the derivation of nine hESC at the Barcelona Stem Cell Bank in the Center for Regenerative Medicine in Barcelona.

\section{Materials and methods}

Source of embryos. Human embryos were donated for research by couples who submitted to in vitro fertilization (IVF) cycles at different assisted reproduction centers (Institut Universitari Dexeus and Fundació Puigvert in Barcelona and Clinica Girona in Girona). Embryos were used for the present study after the informed consent of the couples, the approval of the Ethics Committee, and the approval of the Comisión de Seguimiento y Control de la Donación de Células y Tejidos Humanos del Instituto de Salud Carlos III. Derivations were performed at the Stem Cell Bank of the Center for Regenerative Medicine in Barcelona.

Embryos were thawed using Vitrolife thawing media (Thaw-kit 1 or Thaw-kit Blast, Vitrolife Sweden AB, Göteborg, Sweden) depending on the embryo stage and according to the manufacturer's instructions.

Derivation of hESC lines. Early cleavage-thawed embryos were cultured in G1.2 (Vitrolife) until day 3 and cultured in
G2.2 (Vitrolife) to the blastocyst stage. Embryos thawed at blastocyst stage were cultured in G2.2 overnight for reexpansion. Blastocysts were classified according to the classification proposed by Stephenson et al. (2006), taking into account the expansion degree, the number of cells and appearance of the inner cell mass (ICM), and the number of cells and cohesion of the trophectoderm. The zona pellucida was removed with $5 \mathrm{mg} / \mathrm{ml}$ pronase (Roche) or with Tyrode's acid (Medicult, Barcelona, Spain). Fragments and degenerated cells were gently removed with a fire sharp Pasteur pipette (Humagen, Charlottesville, VA). In the initial phase of derivation, immunosurgery for ICM isolation was performed in good quality blastocysts. These blastocysts were incubated in antihuman whole serum antibody (1:4; Sigma, St. Louis, MO) for $30 \mathrm{~min}$ followed by exposure to guinea pig complement $(1: 4$; Sigma) for $30 \mathrm{~min}$ more. The trophectoderm was removed with a firesharpened Pasteur pipette (Humagen).

In order to avoid the use of antibodies and complement from animal origin, immunosurgery was not performed in a second phase, and whole blastocysts were seeded, especially when poor quality blastocysts were used.

Human foreskin fibroblasts (HFF-1, CCD1112Sk ATCC, Manassas, VA) were used as feeders for the ICM's or blastocyst culture and were prepared as follows: The medium used for feeder cell culture was Iscove's Modified Dulbecco's Medium (Invitrogen, Carlsbad, CA) supplemented with $10 \%$ fetal bovine serum (FBS; Gibco, Carlsbad, $\mathrm{CA}$; Invitrogen) and $0.5 \%$ penicillin-streptomycin (Gibco, Invitrogen). HFF were irradiated at $55 \mathrm{~Gy}$ and plated in $0.1 \%$ gelatine-coated IVF dishes (Falcon, Becton Dickinson, Franklin Lakes, NJ; $7 \times 10^{4} / \mathrm{cm}^{2}$; Hovatta et al., 2003). Whole blastocysts or isolated ICM's were transferred on feeder's dishes and cultured at $37^{\circ} \mathrm{C}$ and $5 \% \mathrm{CO}_{2}$ in KnockOut Dulbecco's modified Eagle's medium (KODMEM) supplemented with $2 \mathrm{mmol} / \mathrm{l}$ GlutaMAX (Gibco, Invitrogen), $0.05 \mathrm{mmol} / 1$ 2-mercaptoethanol (Gibco, Invitrogen), $8 \mathrm{ng} / \mathrm{ml}$ basic fibroblast growth factor (Invitrogen), $1 \%$ nonessential amino acids (Cambrex, East Rutherford, NJ), 20\% KnockOut Serum Replacement (SR; Invitrogen), and $0.5 \%$ penicillin-streptomycin (Gibco, Invitrogen). After 4-5 d in culture, a small clump of cells with hESC
Table 1. Results of embryo thawing and hESC derivation (first series)

\begin{tabular}{lllll}
\hline 1st series & 2PN zygotes & Cleavage stage embryos & Blastocysts & Total \\
\hline Embryos thawed & 20 & 35 & 6 & 61 \\
Embryos surviving (\%) & $10(50 \%)$ & $28(80 \%)$ & $4(66.7 \%)$ & $42(68.8 \%)$ \\
Blastocysts (\%) & $3(30 \%)$ & $10(35.7 \%)$ & & 17 \\
Blastocyst/ICM seeded & 3 & 10 & 4 & 5 \\
Outgrowths & - & 3 & 2 & $5(29.4 \%)$ \\
hESC lines & 0 & $3(30 \%)$ & $2(50 \%)$ & \\
\hline
\end{tabular}


Figure 1. $A$ and $B$ Initial

outgrowths of the nine cell lines:

ES [2], ES [3], ES [4], ES[5], ES

[6], ES[7], ES[8], ES[9], and

$E S[10]$. $C$ colonies at passage 1 .

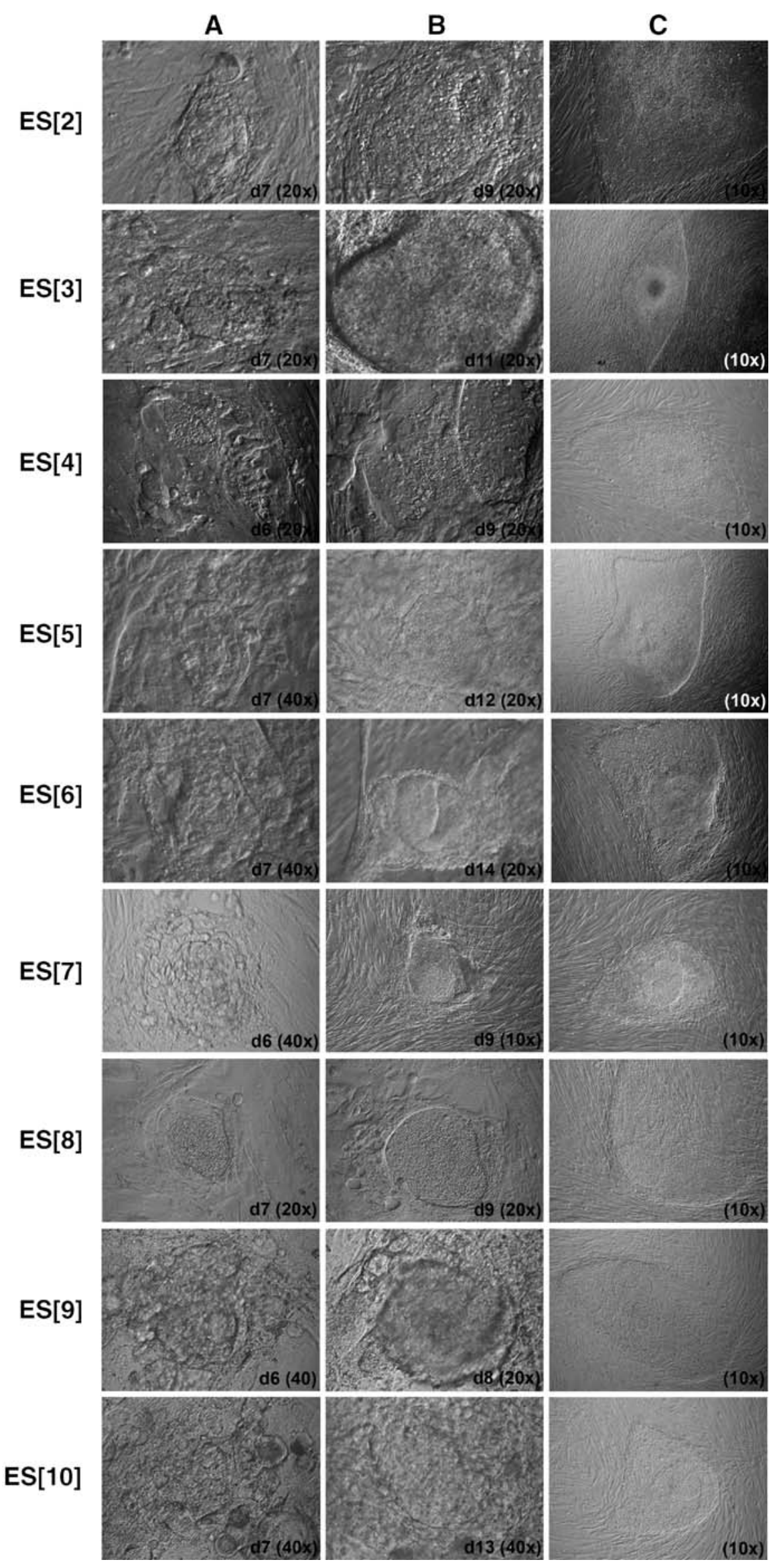


Table 2. Blastocyst quality (Stephenson et al. 2006)

\begin{tabular}{ll}
\hline Embryo classification & Line \\
\hline $2 \mathrm{DC}$ & ES2 \\
$3 \mathrm{CC}$ & ES3 \\
$1 \mathrm{DC}$ & ES4 \\
$2 \mathrm{BA}$ & ES5 \\
$2 \mathrm{DB}$ & ES6 \\
$2 \mathrm{DC}$ & ES7 \\
Compacted embryo & ES8 \\
$3 \mathrm{BB}$ & ES9 \\
$3 \mathrm{BB}$ & ES10 \\
\hline
\end{tabular}

Scoring blastocyst quality according Stephenson criteria:

Expansion status: (1) no expansion in overall size, zona pellucida still thick; (2) some expansion in overall size, zona pellucida beginning to thin; (3) full expansion, zona pellucida very thin; (4) partially hatched, no expansion in overall size: seen following preimplantation genetic diagnosis; (5) fully hatched blastocysts, zona pellucida may contain cells rejected during blastocyst formation.

ICM appearance: (1) cells compacted, tightly adhered together and indistinguishable as individual cells; (2) cells less compacted so larger in size, loosely adhered together, some visible as individual cells; (3) very few cells visible, either compacted or loose may be difficult to completely distinguish from trophectoderm; (4) cells of ICM appear degenerate; (5) no ICM cells visible in any plane

Trophectoderm appearance: (1) many small identical cells forming a continuous trophectoderm layer; (2) Fewer, larger cells, may not form completely continuous layer; (3) sparse cells, may be very large, very flat or appear degenerate.

morphology (a compact colony structure, a high ratio of nucleus to cytoplasm, and prominent nucleoli) appeared among other cell types and unspecific growth. The colony growth was checked and the medium changed daily until around day 10-12 when the initial colony was mechanically dissociated into small clumps using a $150-\mu \mathrm{m} \varnothing$ plastic pipette (The Stripper, Midatlantic Diagnostics, Medford, NJ) and replated on a new feeder layer and fresh medium. From then on, every fifth to seventh $d$, individual undifferentiated colonies were mechanically dissociated into small clumps and replated. Two of the cell lines were adapted from early passages to feeder-free culture. The cells were dissociated using trypsin $(0.05 \%$ trypsin/EDTA, Invitrogen) and plated on Matrigel (Becton Dickinson; diluted 1:15 with KO-DMEM) in mouse embryonic fibroblasts (MEFs; from 13,5 C57BL/6 mouse embryos) conditioned hES medium.

Recently and in order to improve our results, some methodological modifications were made (Chen et al. 2009). With the objective of increasing the derivation efficiency, different strategies were carried out depending on embryo quality. Poor quality embryos were cultured from day 5 , for 2-3 d, in conditioned medium (hES medium exposed to growing culture of hESC for $1 \mathrm{~d}$ with $2.5 \%$ of ES cell tested FBS, Hyclone), to try to stimulate ES cells growth in the embryo. The ICM of good quality embryos was isolated by exposing the trophectoderm to cell lethal laser pulses (Octax EyeWare, Olympus, Center Valley, PA). All whole embryos and ICM's were cultured on irradiated HFF monolayers in derivation media (hES medium 1:1 dilution with hES conditioned-medium) supplemented with $2.5 \%$ of ES cell-tested FBS.

Colonies were periodically selected and cryopreserved. They were mechanically split in small clumps and centrifuged at 750-1,000 rpm for $1 \mathrm{~min}$. The supernatant was aspirated, and the pellet was gently mixed with freezing medium (90\% FBS $+10 \%$ DMSO), precooled

Table 3. ES[2], ES[3], ES[4], ES[5], and ES[6] characterization

\begin{tabular}{|c|c|c|c|c|c|}
\hline & $\mathrm{ES}[2]$ & $\mathrm{ES}[3]$ & $\mathrm{ES}[4]$ & $\mathrm{ES}[5]$ & $\mathrm{ES}[6]$ \\
\hline Karyotype & $\begin{array}{l}46, \\
\mathrm{XY}\end{array}$ & $\begin{array}{l}\text { 46, } \\
\mathrm{XY}\end{array}$ & $\begin{array}{l}46, \\
\mathrm{XY}\end{array}$ & $\begin{array}{l}\text { 46, } \\
\mathrm{XY}\end{array}$ & $\begin{array}{l}\text { 46, } \\
\mathrm{XY}\end{array}$ \\
\hline Passage & 21 & 25 & 17 & 19 & $20 / 97$ \\
\hline $\begin{array}{l}\text { Alkaline } \\
\text { phosphatase }\end{array}$ & + & + & + & + & + \\
\hline Passage & 9 & 9 & 20 & 18 & 22 \\
\hline Telomerase & + & + & + & + & + \\
\hline Passage & 27 & 27 & 28 & 22 & 58 \\
\hline HLA & Done & Done & Done & Done & Done \\
\hline Passage & 17 & 17 & 37 & 19 & 39 \\
\hline \multicolumn{6}{|c|}{ Pluripotency markers } \\
\hline Passage & 17 & 15 & 24 & 16 & 21 \\
\hline SSEA-3 & + & + & + & + & + \\
\hline SSEA-4 & + & + & + & + & + \\
\hline TRA-1-60 & + & + & + & + & + \\
\hline TRA-1-81 & + & + & + & + & + \\
\hline Oct-4 & + & + & + & + & + \\
\hline NANOG & + & + & + & + & + \\
\hline SOX-2 & + & + & + & + & + \\
\hline Freezing/thawing & + & + & + & + & + \\
\hline \multicolumn{6}{|l|}{ Pluripotency } \\
\hline \multicolumn{6}{|l|}{$\begin{array}{l}\text { In vitro (EB } \\
\text { formation) }\end{array}$} \\
\hline Passage & 17 & $17 / 99$ & 26 & 20 & 42 \\
\hline Endoderm & + & + & + & + & + \\
\hline Ectoderm & + & + & + & + & + \\
\hline Mesoderm & + & + & + & + & + \\
\hline \multicolumn{6}{|c|}{ In vivo (teratomas) } \\
\hline Passage & 26 & 26 & 17 & 26 & 46 \\
\hline Endoderm & + & + & + & + & + \\
\hline Ectoderm & + & + & + & + & + \\
\hline Mesoderm & + & + & + & + & + \\
\hline
\end{tabular}




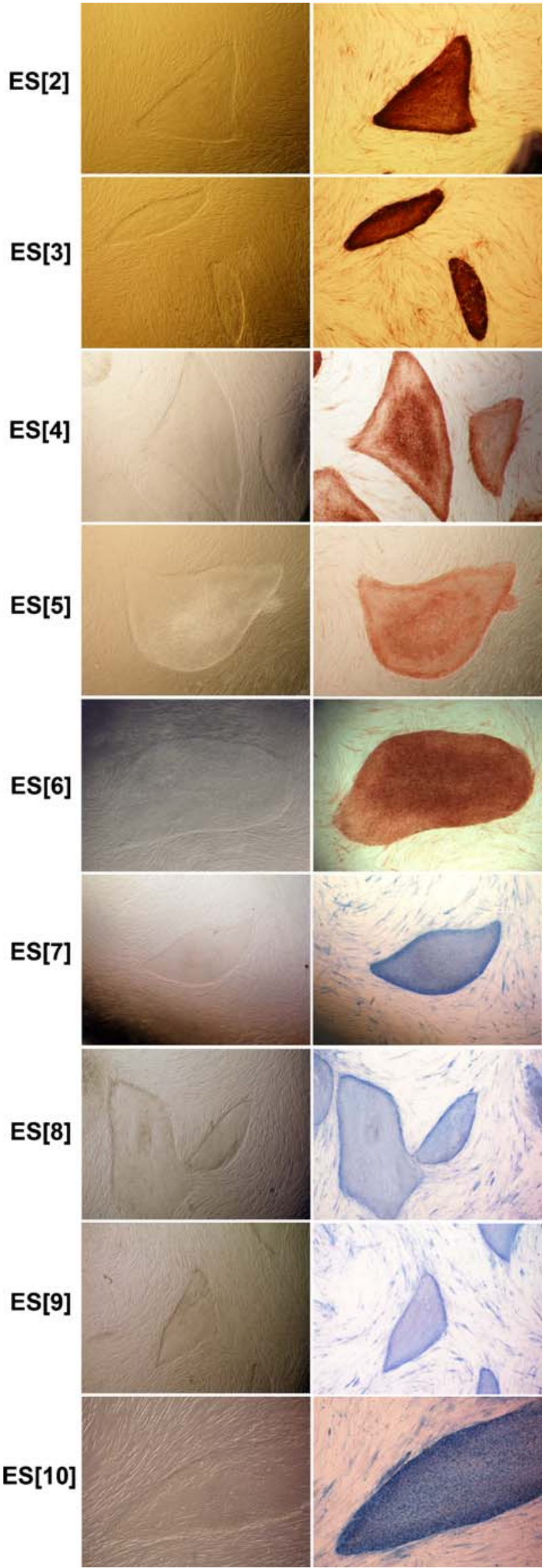

Figure 2. Analysis of the alkaline phosphatase activity in the five lines, $E S[2]$ to $E S[10]$.

on ice, and stored in cryotube vials (NUNC, Roskilde, Denmark). A programmable freezer was used with a cooling rate of $0.5^{\circ} \mathrm{C} / \mathrm{min}$. The seeding was induced at $-10^{\circ} \mathrm{C}$. Then the cooling rate continued at $-0.5^{\circ} \mathrm{C} / \mathrm{min}$ until $-35^{\circ} \mathrm{C}$, and then the cryovials were transferred directly in liquid nitrogen.

After thawing at $37^{\circ} \mathrm{C}$, colonies were plated on new feeder layers in $\mathrm{hES}$ medium to assess freezing/thawing survival.

Characterization of hESC. To determine if these lines were composed of hESC, phenotypic and genetic analysis of undifferentiated colonies was performed. This characterization included karyotype, analysis of human leukocyte antigen (HLA), assessment of expression of pluripotency markers, and evaluation of pluripotency in vitro and in vivo. To assess pluripotency markers, immunocytochemistry was used, with the following antibodies at the indicated dilutions: mouse anti-OCT4 (1:500, Santa Cruz Biotechnology, Santa Cruz, CA), rabbit anti-SOX2 (1:100, Chemicon, Temecula, CA), rabbit anti-Nanog (1:500, Abcam, Cambridge, UK), rat anti-SSEA-3, mouse anti-SSEA-4, mouse anti-TRA-1-60, mouse anti-TRA-1-81 (1:10, Chemicon). Incubation with primary antibody was performed for $24 \mathrm{~h}$ at $4^{\circ} \mathrm{C}$. Incubation with FITC, Cy2, or Cy3 conjugate secondary antibodies $(1 ; 200$, Jackson ImmunoResearch, West Grove, PA) was made for $2 \mathrm{~h}$ at RT followed by the counterstaining with DAPI $(10 \mathrm{mg} / \mathrm{ml}$, Sigma $)$. Alkaline phosphatase activity was measured by using the Alkaline Phosphatase Red and Blue Membrane Substrate (Sigma) after fixation 2 min with 4\% paraformaldehyde (PFA). Karyotyping was performed by incubating colonies for $30 \mathrm{~min}$ in $2 \mu \mathrm{l} / \mathrm{ml}$ colcemid (Invitrogen) and by using the G-banding method. Fifteen metaphases were analyzed for each line. Telomerase activity was detected using TRAPeze Telomerase Detection Kit (Chemicon International). HLA was analyzed to determine histocompatibility by sequence-based typification with the AlleleSEQR HLA Sequencing Kit (Atria Genetics, San Francisco, CA). Analysis of microsatellites was performed by multiplex PCR of nine microsatellites or short tandem repeats (STRs) plus amelogenine gene using AmplFiSTR Profiler Plus Kit (Applied Biosystems, Foster City, CA). The pluripotency of the hESC lines was tested both in vitro and in vivo. For in vitro differentiation, embryoid bodies (EBs) were generated by mechanical pick-up of whole colonies. Colonies were then cultured in nonadherent dishes for 3-4 $\mathrm{d}$ in $\mathrm{hES}$ medium. Embryoid bodies were then plated in $0.1 \%$ 
gelatine-coated glass chamber slides and cultured in DMEM supplemented with 20\% KnockOut SR (Gibco/ BRL, Invitrogen), 0,1 mM B-mercaptoethanol, 1\% nonessential amino acids (GIBCO/BRL) for $15-25 \mathrm{~d}$ before fixing for 15 min with $4 \%$ PFA. To induce mesodermal differentiation, EBs were cultured in EB medium in the presence of $10 \mu \mathrm{mol} / \mathrm{L}$ ascorbic acid (Sigma) for $12-28 \mathrm{~d}$ (Raya et al. 2008). hESC neuronal differentiation was obtained by coculture with PA6 cells for $3-5$ wk under the following differentiation conditions: DMEM/F12 Glutamax (GIBCO), 10\% KnockOut Serum Replacement (GIBCO), $0.1 \mathrm{mM}$ nonessential amino acids (GIBCO), and $0.1 \mathrm{mM}$ 2-mercaptoethanol (GIBCO; Kitajima et al. 2005). To assess the expression of markers associated with differentiation of the three embryonic germ layers, cells were stained with the primary antibody $\alpha$-smooth muscle actin (1:400, Sigma), $\alpha$-sarcomeric-actinin (1:100, Sigma), GATA 4 (1:50, SantaCruz), anti- $\beta$-tubulin III (1:1000, Covance, Harrogate, UK), GFAP (1:1000, Dako, Carpinteria, CA), FoxA2 (1:50, R\&D Systems, Minneapolis, MN), mouse and rabbit anti- $\alpha$-fetoprotein (1:400, Dako) as markers of mesoderm, ectoderm, and endoderm, respectively. The pluripotency in vivo was shown by the generation of teratomas via intramuscular injection of approximately one million of cells into severely combined immunodeficient (SCID) mice. Eight weeks after, mice were sacrificed, and tumors were analyzed with hematoxylin and eosin staining and conventional immunohistochemistry techniques.

\section{Results and Discussion}

In the first series, 61 embryos were thawed. The mean survival rate was $68.8 \%, 50 \%$ for pronuclear stage zygotes, $80 \%$ for cleavage stage embryos, and $67 \%$ for blastocyst stage embryos (Table 1). From the 38 embryos surviving at early cleavage stages, 13 reached the blastocyst stage. The blastocyst rate was $34.2 \%$. Seventeen embryos were seeded, and five cell lines were obtained (ES[2]-ES[6]; Fig. 1), all of them from whole blastocyst seeding. The derivation efficiency was $29.4 \%$. Nine embryos did not have a clear ICM and had degenerated areas, and some did not show clear blastocyst morphology at the seeding. Only few cells were morphologically viable in some of the embryos. All blastocysts were classified according to Stephenson's proposal (Stephenson et al. 2006), taking into account the expansion status, the number of cells and cohesion of the ICM, and the number of cells and appearance of trophectoderm. ES[2] and ES[4] lines were derived from two blastocysts (midexpanded and nonexpanded poor quality ICM blastocysts, respectively, and

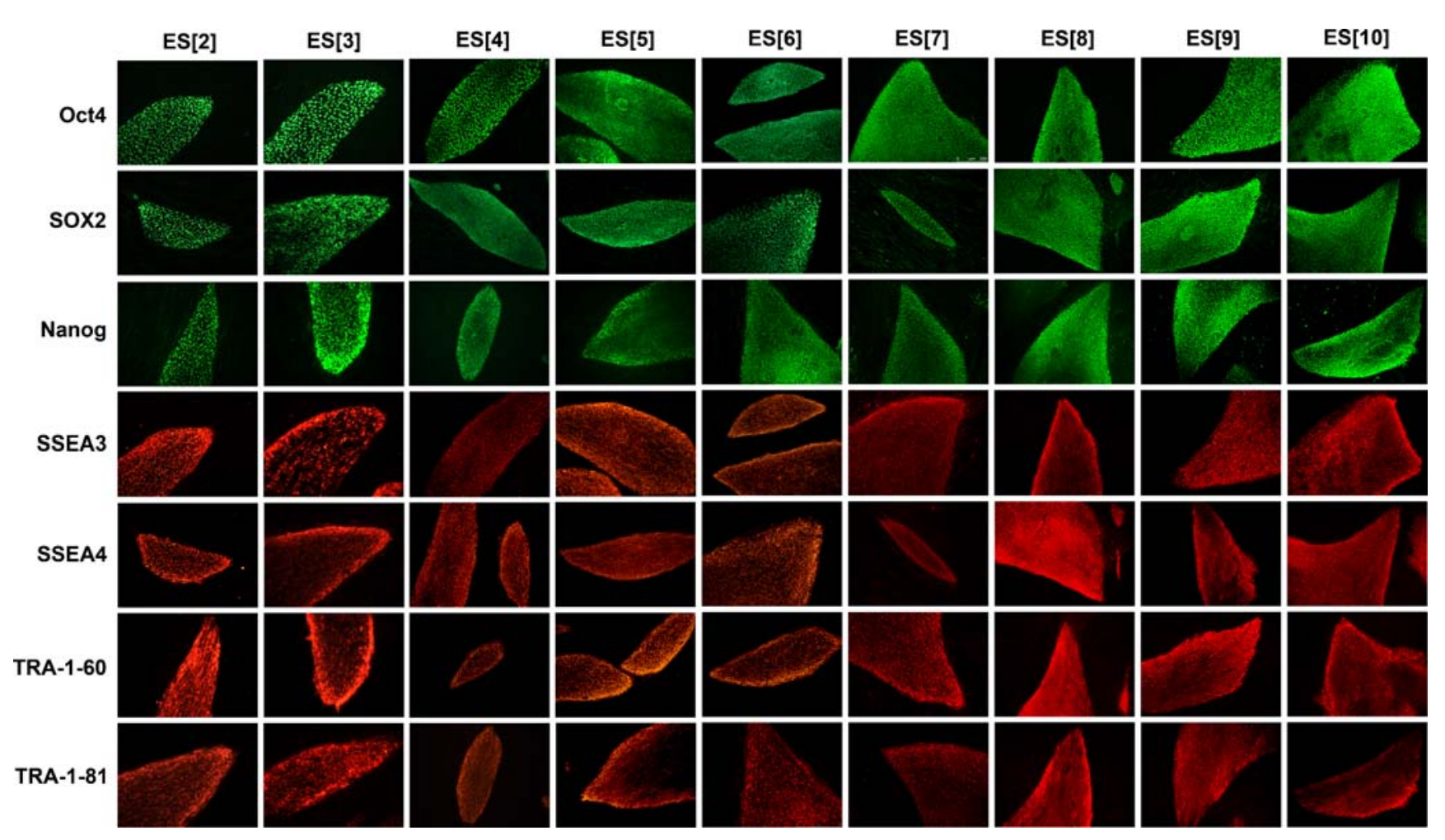

Figure 3. Expression of pluripotency markers SSEA3, SSEA4, TRA-1-60, TRA-1-81, Oct4, SOX2, and Nanog in lines ES[2] to ES[10]. 
Figure 4. In vitro differentiation to the three germ layers. Ectoderm: $\beta$-tubulin III (ES[3] and ES[9] show also GFAP in red). Endoderm: $\alpha$-feto-proteina (ES[6], ES[7], ES[8], ES[9], and ES[10] show also FOXA2 in red). Mesoderm: $\alpha$-smooth muscle actin ES[2], ES[7], and ES[9]; $\alpha$-sarcomeric-actinin, ES [3], ES[4], ES [5], ES[6], and $E S[8]$ (ES[6], ES[8], and ES[10] show also GATA 4 in green).

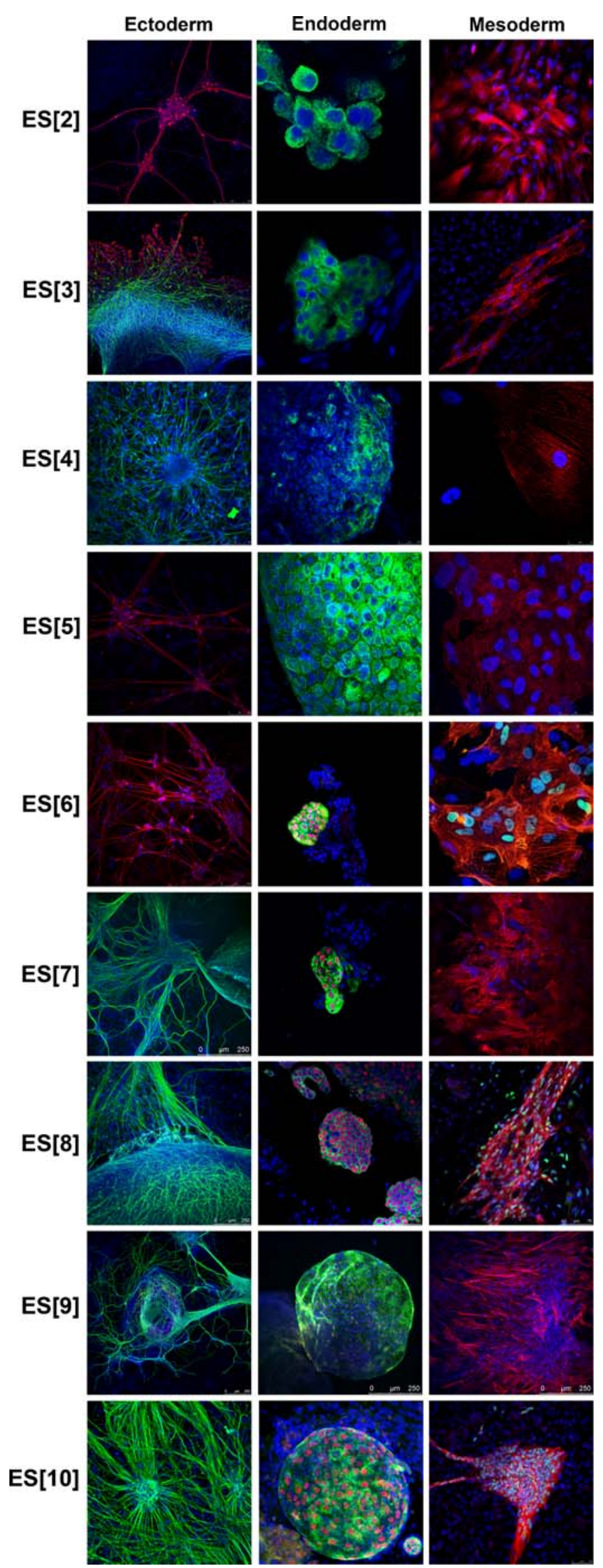



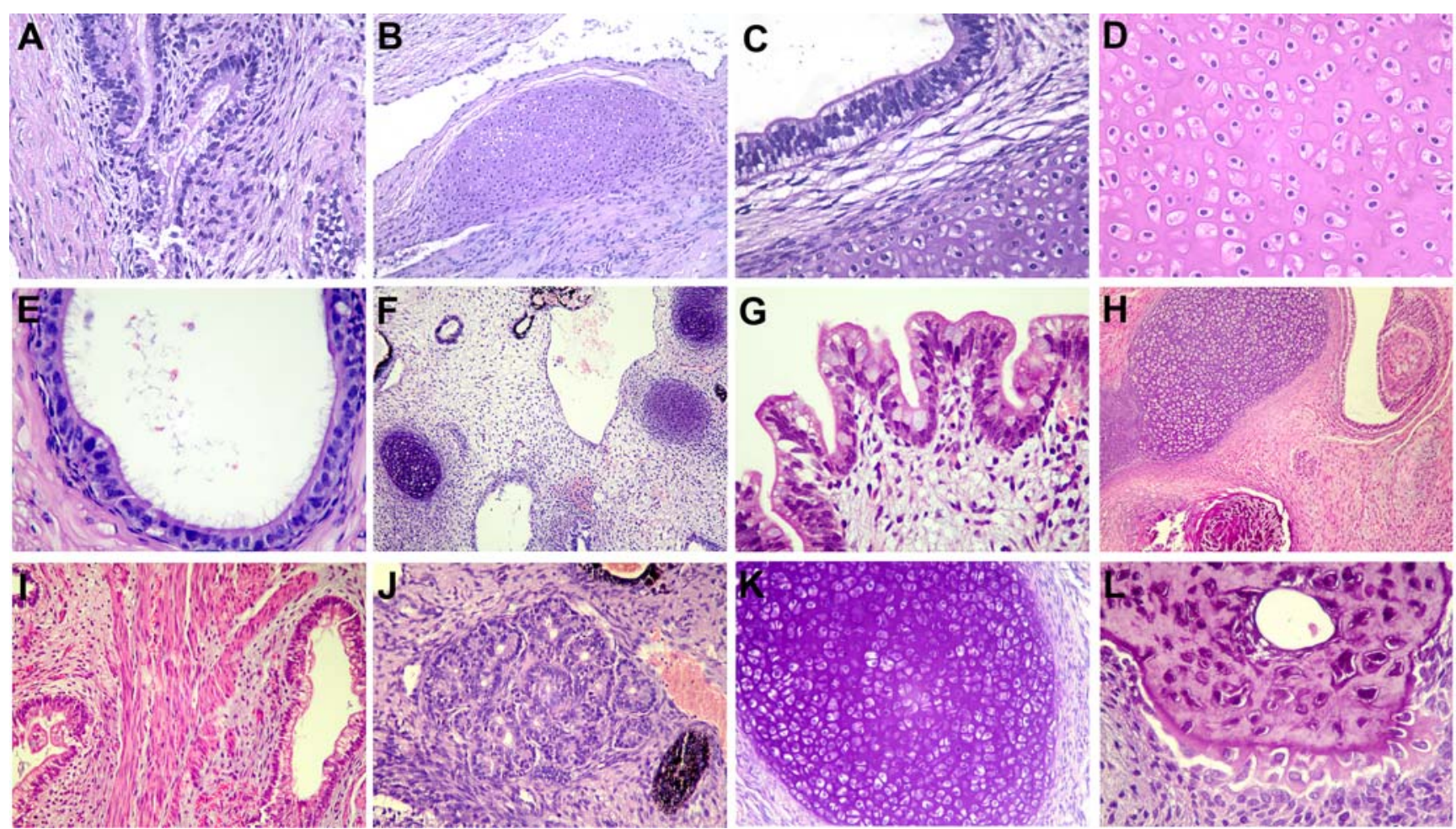

Figure 5. In vivo differentiation. (a) Respiratory epithelia ES[2]. (b) Cartilage ES[2]. (c) Respiratory epithelia ES[3]. (f) Cartilage ES[3]. (e) Ciliated epithelia ES[4]. (f) Cartilage, epithelia, and mesenchymal

structures ES[4]. (g) Secreting epithelia ES[5]. (h) Cartilage, bone, and epithelia tissue ES[5]. (i) Smooth muscle ES[5]. (j) Cartilage ES[6]. (k) Glandular tissue ES[6]. (l) Bone tissue ES[6].

sparse trophectoderm cells 2DC, 1DC). ES[6] was obtained from a midexpanded blastocyst, with the cells of ICM cells showing degeneration signs and with the trophectoderm not forming a continuous layer (2DB). ES[3] line was derived from a fully expanded blastocyst, with a visible ICM with few cells and sparse cells in the trophectoderm (3CC). ES[5] comes from a midexpanded blastocyst, showing ICM compacted cells, some of them visible as individual cells and a trophectoderm forming a continuous layer with many small identical cells (2BA; Table 2). ES[4] was adapted from passage 3 to feeder-free culture on Matrigel and MEF's conditioned

ES[2]

ES[3] $\quad$ ES[4]

ES[5]

ES[6]

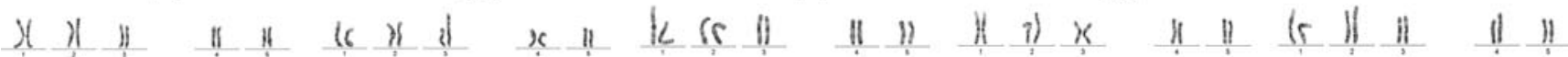

I

"

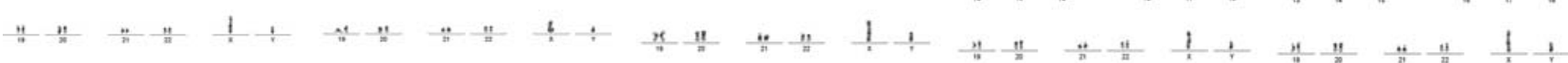

ES[7]

$\mathrm{ES}[8]$

ES[9]

ES[10]

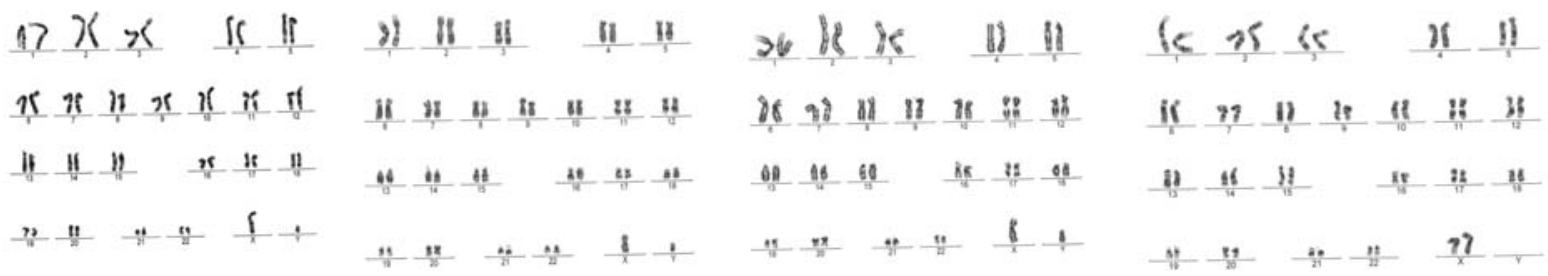

Figure 6. Karyotypes of the nine lines ES[2] to ES[10]. 
Table 4. Results of embryo thawing and hESC derivation (second series)

\begin{tabular}{ll}
\hline 2nd series & Cleavage stage embryos \\
\hline Embryos thawed & 66 \\
Embryos surviving (\%) & $58(87.8 \%)$ \\
Blastocysts (\%) & $13(22.4 \%)$ \\
Blastocyst/ICM seeded & 11 \\
Outgrowths & 1 \\
hESC lines & $1(9.1 \%)$ \\
\hline
\end{tabular}

medium and maintained in this condition for more than 30 passages.

The five lines were characterized. A summary of the characterization performed is presented in Table 3. All of them were positive for all the pluripotency markers we have tested. They express alkaline phosphatase (Fig. 2), Oct4, Nanog, SOX2, SSEA-3, SSEA-4, TRA-1-60, and TRA-1-81 (Fig. 3). They also showed telomerase activity (data not shown). Generated EB's from the five lines expressed markers of ectoderm ( $\beta$-tubulin), endoderm $(\alpha$-fetoprotein), and mesoderm ( $\alpha$-actinin) after in vitro culture (Fig. 4). The supplementation of the differentiation medium with ascorbic acid resulted in approximately $20 \%$ of the embryoid bodies generated displaying rhythmically beating areas in the five lines. The ectoderm differentiation with PA6 coculture protocol yielded a high percentage of neurons.

All of them had potential to form teratomas in SCID mice with presence of tissues from the three germ layers as cartilage, respiratory epithelium, and neuronal tissue (Fig. 5). All five lines showed normal karyotype (46, XY; Fig. 6). Table 7 shows the HLA typing results. All of them were different except for ES[2] and ES[3] which came from two embryos from the same couple. The microsatellites analysis for ES[2] and ES[3] were different and compatible with the fact that the embryos were from the same progenitor couple (data not published but provided to the reviewers).

The five lines were registered in the Spanish Stem Cell Bank and the hESCreg (www.isciii.es; www.hescreg.eu). All cell lines are over 20 passages (one of them is at passage 122 at the time of writing), and adequate frozen stocks are available. These lines are provided at scientist's request without cost (except for freezing and shipping expenses) with the project approval by the competent authority (Comisión de Seguimiento y Control de la Donación de Células y Tejidos Humanos del Instituto de Salud Carlos III).
A new hESC line, at present in passage 21, was derived from the 2 nd series of 66 thawed embryos. In this series, 58 embryos survived to the thawing process $(87.8 \%)$, and 13 reached the blastocyst stage (22.4\%; Table 4). Eleven whole blastocysts were seeded, and one line was obtained (ES[7]; Fig. 1). The derivation efficiency was 9.1\%. ES[7] was derived from a partially degenerated embryo with only few surviving cells (2DC; Table 2). ES[7] was adapted from passage 3 to feeder-free culture on Matrigel and MEF's conditioned medium.

In a new series (third series), only 15 embryos survived the freezing and thawing process out of 62 thawed embryos (survival rate $24.2 \%$ ). From these, four embryos reached the blastocyst stage (blastocyst rate 26.7\%). One whole blastocyst was seeded, and three ICM's were isolated by laser before seeding. One compacted embryo was also seeded after $2 \mathrm{~d}$ of culture in hES-conditioned medium. Three lines were obtained (ES[8]-ES[10]; Fig. 1). The derivation efficiency was $60 \%$ (Table 5). ES[8] comes from the compacted embryo. ES[9] and ES[10] were obtained from expanded blastocysts (3BB) with laser-isolated ICM's (Table 2).

Characterization of the above mentioned four hESC is also completed; only results of differentiation in vivo are pending. Table 6 shows a summary of the characterization of these lines. Alkaline phosphatase expression and others pluripotency markers (Oct4, Nanog, SOX2, SSEA-3, SSEA-4, TRA-1-60, and TRA-1-81) are shown in Figs. 2 and 3, respectively. Figure 4 shows expressed markers of ectoderm ( $\beta$-tubulin), endoderm ( $\alpha$-fetoprotein or FoxA2), and mesoderm ( $\alpha$-actinin or GATA4) after in vitro culture. Lines ES[7] to ES[9] showed normal male karyotypes (46, $\mathrm{XY}$ ), while ES[10] is female (46, XX; Fig. 6). Table 7 shows HLA typing results.

All lines were tested for mycoplasma routinely every month. The method used was VenorGeM Mycoplasma detection kit for conventional PCR (Minerva Biolabs, Berlin, Germany). The lines were also tested for bacteria (aerobic and anaerobic) and fungi.

Table 5. Results of embryo thawing and hESC derivation (third series)

\begin{tabular}{ll}
\hline 3rd series & Cleavage stage embryos \\
\hline Embryos thawed & 62 \\
Embryos surviving (\%) & $15(24.2 \%)$ \\
Blastocysts (\%) & $4(26.7 \%)$ \\
Blastocyst/ICM seeded & 5 \\
Outgrowths & 3 \\
hESC lines & $3(60 \%)$ \\
\hline
\end{tabular}


Table 6. ES[7], ES[8], ES[9], and ES[10] characterization

\begin{tabular}{|c|c|c|c|c|}
\hline & ES[7] & $\mathrm{ES}[8]$ & $\mathrm{ES}[9]$ & $\mathrm{ES}[10]$ \\
\hline Karyotype & $46, \mathrm{XY}$ & $46, \mathrm{XY}$ & $46, \mathrm{XY}$ & $46, \mathrm{XX}$ \\
\hline Passage & 9 & 10 & 10 & 10 \\
\hline Alkaline phosphatase & + & + & + & + \\
\hline Passage & 21 & 14 & 15 & 14 \\
\hline HLA & Done & Done & Done & Done \\
\hline passage & 10 & 10 & 10 & 11 \\
\hline \multicolumn{5}{|l|}{ Pluripotency markers } \\
\hline Passage & 10 & 10 & 10 & 10 \\
\hline SSEA-3 & + & + & + & + \\
\hline SSEA-4 & + & + & + & + \\
\hline TRA-1-60 & + & + & + & + \\
\hline TRA-1-81 & + & + & + & + \\
\hline Oct-4 & + & + & + & + \\
\hline NANOG & + & + & + & + \\
\hline SOX-2 & + & + & + & + \\
\hline \multicolumn{5}{|l|}{ Pluripotency } \\
\hline \multicolumn{5}{|c|}{ In vitro (EB formation) } \\
\hline Passage & 10 & 10 & 10 & 12 \\
\hline Endoderm & + & + & + & + \\
\hline Ectoderm & + & + & + & + \\
\hline Mesoderm & + & + & + & + \\
\hline In vivo (teratomas) & On going & On going & On going & On going \\
\hline Passage & 24 & 12 & 11 & 15 \\
\hline
\end{tabular}

Table 7. HLA results of ES[2]-ES[10]

\begin{tabular}{|c|c|c|c|c|c|}
\hline & HLA-A & HLA-B & HLA-C & HLA-DRB & HLA-DQB \\
\hline \multirow[t]{2}{*}{$\mathrm{ES}[2]$} & A 0101 & В 0801 & Cw 0701 & DRB1 0301 & DQB1 0201 \\
\hline & A 0101 & В 0801 & Cw 0701 & DRB1 0301 & DQB1 0201 \\
\hline \multirow[t]{2}{*}{$\mathrm{ES}[3]$} & A 0101 & В 0801 & Cw 0701 & DRB1 0301 & DQB1 0201 \\
\hline & A 0101 & В 0801 & Cw 0701 & DRB1 0301 & DQB1 0201 \\
\hline \multirow[t]{2}{*}{$\mathrm{ES}[4]$} & A 0201 & B 1402 & Cw 0501 & DRB1 1301 & DQB1 0301 \\
\hline & A 3201 & B 4402 & Cw 0802 & DRB1 1303 & DQB1 0603 \\
\hline \multirow[t]{2}{*}{$\mathrm{ES}[5]$} & A 0301 & B 2702 & Cw 0202 & DRB1 0405 & \\
\hline & A 2402 & В 4901 & Cw 0701 & DRB1 1101 & \\
\hline \multirow[t]{2}{*}{$\mathrm{ES}[6]$} & A 0101 & В 1501 & Cw 0202 & DRB1 1101 & DQB1 0301 \\
\hline & A 2402 & B 2702 & Cw 0303 & DRB1 1301 & DQB1 0603 \\
\hline \multirow[t]{2}{*}{$\mathrm{ES}[7]$} & A 03 & B 35 & $\mathrm{Cw} 04$ & DRB1 01 & DQB1 02 \\
\hline & A 11 & B 47 & $\mathrm{Cw} 06$ & DRB1 07 & DQB1 05 \\
\hline \multirow[t]{2}{*}{$\mathrm{ES}[8]$} & A 02 & В 18 & $\mathrm{Cw} 07$ & DRB1 07 & DQB1 02 \\
\hline & - & B 44 & $\mathrm{Cw} 16$ & DRB1 08 & DQB1 04 \\
\hline \multirow[t]{2}{*}{ ES[9] } & A 02 & B 14 & $\mathrm{Cw} 08$ & DRB1 03 & DQB1 02 \\
\hline & A 33 & B 51 & $\mathrm{Cw} 14$ & DRB1 15 & DQB1 06 \\
\hline \multirow[t]{2}{*}{$\mathrm{ES}[10]$} & A 02 & B 18 & $\mathrm{Cw} 12$ & DRB1 07 & DQB1 02 \\
\hline & A 25 & B 44 & $\mathrm{Cw} 16$ & DRB1 05 & DQB1 05 \\
\hline
\end{tabular}


Acknowledgments The Stem Cell Bank at the Center for Regenerative Medicine in Barcelona wants to express its gratitude to Institut Universitari Dexeus, Fundació Puigvert and Clínica Girona for providing the embryos used in the derivations.

I.R-P. is a recipient of a MEC predoctoral fellowship. This work was partially supported by Red de Terapia Celular (Tercel) and Fundación Cellex grant (to JC.I.).

\section{References}

Chen A.; Egli D.; Niakan K.; Deng J.; Akutsu H.; Yamaki M. et al. Optimal timing of inner cell mass isolation increases the efficiency of human embryonic stem cell derivation and allows generation of sibling cell lines. Cell Stem Cell 4: 103-106; 2009.

Hovatta O.; Mikkola M.; Gertow K.; Stromberg A. M.; Inzunza J.; Hreinsson J.; Rozell B.; Blennow E.; Andang M.; AhrlundRichter L. A culture system using human foreskin fibroblasts as feeder cells allows production of human embryonic stem cells. Hum. Reprod. 18: 1404-1409; 2003.
Kitajima H.; Yoshimura S.; Kokuzawa J.; Kato M.; Iwama T.; Motohashi T.; Kunisada T.; Saki N. Culture method for the induction of neurospheres from mouse embryonic stem cells by coculture with PA6 stromal cells. J. Neurosci. Res 80: 467-474; 2005.

Ley 45/2003, de 21 de Noviembre, por la que se modifica la Ley 35/ 1988 de 22 de Noviembre, sobre Técnicas de Reproducción Humana Asistida.

Ley 14/2006, de 26 de Mayo, sobre Técnica de Reproducción Humana Asistida

Ley 14/2007 de 3 de Julio, de Investigación Biomédica.

Orden SCO/393/2006, de 8 de febrero, por la que se establece la organización y funcionamiento del Banco Nacional de Líneas Celulares

Raya A.; Rodríguez-Pizà I.; Aran B.; Consiglio A.; Barri P. N.; Veiga A.; Izpisúa J. Generation of cardiomyocytes from new human embryonic stem cell lines derived from poor-quality blastocysts. Cold Spring Harbor Symposia on Quantitative Biology 73: 2735; 2008.

Stephenson E. L.; Braude P. R.; Mason C. Proposal for a universal minimum information convention for the reporting on the derivation of human embryonic stem cell lines. Regen. Med. 1: 739-750; 2006. 